\title{
The role of intravelar veloplasty in primary cleft palate repair
}

\section{Abstract}

Background and objective: The presence of a cleft palate introduces feeding difficulties, problems in speech development, and the possibility of impaired facial growth. Intravelar veloplasty aids to gain functional repair of the palate. This study aimed to evaluate the effect of intravelar veloplasty on the function of velum in primarily repaired palates.

Methods: A review study was done in Rizgary Teaching Hospital, Erbil. Clinical and nasopharyngeal endoscopic examination was done for patients who underwent primary palatal repair with intravelar veloplasty between 2009 to 2014. The study assessed the velopharyngeal port for adequacy of closure, hypernasality of speech, and oronasal fistula rate.

Results: This study included 47 patients. The mean age of patients \pm SD was $3.4 \pm 4$ years (range 1- 20 years) at time of repair. Major improvement in intelligibility and production of pharyngeal sounds and velopharyngeal closure was noticed when using intravelar veloplasty in palatal repair. There was a significant association between defect type and degree of velopharyngeal port closure $(P=0.009)$, i.e., inadequate velopharyngeal closure was more common in the complete cleft palate.

Conclusion: This study showed that intravelar veloplasty helps to improve the velopharyngeal closure and oropharyngeal sounds production. The poor velopharyngeal closure is more likely related to wide, complete cleft palate.

Keywords: Cleft palate; Palatoplasty; Intravelar veloplasty; Velopharyngeal insufficiency.

\section{Introduction}

Cleft lip and palate (CLP) are the most common congenital craniofacial anomalies. The presence of a cleft palate introduces feeding difficulties, concerns regarding speech development, and the possibility of impaired facial growth. The cleft palate (CP) is surgically classified as complete, incomplete, or submucous types. ${ }^{1}$ There are two most commonly used cleft palate repair techniques: a two-flap palatoplasty with intravelarveloplasty (IVV), or Furlow double-opposing z-plasty to achieve the levator repositioning and lengthening of the palate. Both techniques aim to create transverse orientation of the reconstructed levator sling. ${ }^{2}$ Intravelar veloplasty (IVV) is the procedure of reconstruction of the levator muscle sling in CP patients in order to gain velopharyngeal (VP) closure. It is achieved by detaching the levator muscles from their abnormal attachment to the hard palate and repairing them in the midline with the muscle fibers oriented more normally, ${ }^{3}$ transversely rather than vertically. The normal velum consists of the levator muscle in the middle and the tensor aponeurosis anteriorly. While in CP anomaly, the two muscles are closely related, with the tensor aponeurosis attaching to the posterior border of the hard palate and the levator inserting at the margins of the cleft in the anterior half of the velum. ${ }^{1,4}$ Adequate VP closure is essential for the balance of oronasal resonance during speech production. The complete VP closure occurs from the simultaneous movement of the soft palate

* Department of Surgery, College of Medicine, Hawler Medical University, Erbil, Iraq. 
and the lateral and posterior pha-ryngeal walls, which ensures the complete separation between the oral and nasal cavities during the production of oral speech sounds. ${ }^{4}$ Previous anatomic studies of the palatal musculature concluded IVV would decrease the incidence of postpalatoplasty VP insufficiency (VPI). ${ }^{5}$ The term velopharyngeal insufficiency (VPI) refers to a structural deficit in VP sphincter. In this case, there is a communication between the oral and nasal cavities, so, part of the air current is diverged to the nasal cavity during the production of oral speech sounds, leading to the appearance of characteristic symptoms, such as hypernasality. ${ }^{4,6}$ Electromyographic studies demonstrated that lateral pharyngeal wall motion is mainly the result of levator veli palatine muscle contraction and an increase VP competence following primary palatoplasty with IVV had been reported. ${ }^{7}$ Palatoplasty is mostly performed at 6-12 months of age and this provides for early VP competency and the development of normal speech. ${ }^{8,9}$ This study aimed to evaluate the effect of intravelar veloplasty on the function of velum in primarily repaired palates. This study is preliminary in Hawler (Erbil) and it will give us a feedback of late complications rate (oronasal fistula and VPI) in each different cleft palate types and it aims to show the effectiveness of intravelar veloplasty on the functional outcome of palate and on the velopharyngeal port closure.

\section{Methods}

\section{Design and Setting of the Study:}

This is a review of primarily repaired cleft palates with intravelar veloplasty in Erbil governorate from January 2009 to October 2014. The study was conducted in Rizgary Teaching Hospital, Erbil.

\section{The Study Sample:}

Forty seven patients with cleft palate (complete, incomplete, and submucous cleft palate), aged between 1-20 years, who underwent primary palatoplasty, by the same surgeon, were included in the clinical and naso-endoscopic examination. Preoperative preparations were done and after obtaining necessary blood tests, the procedures were performed (palatoplasty with intravelar veloplasty). The exclusion criteria included patients who had secondary palatoplasty, patients who refused to be in the study and patients with associated chronic illness or mental retardation.

\section{The Procedure:}

Different techniques were used for palatolplasty according to the width of cleft but two flaps palatoplasty was most commonly used for our patients. The two flaps palatoplasty ${ }^{10}$ is performed as following; under general anesthesia, one dose of prophylactic antibiotic is given, a Dingman retractor is placed with attention to the endotracheal tube not to be kinked or pulled out of the airway. Lateral incisions are marked just medial to the alveolar ridge, while medially, incisions are also planned along the cleft margin to allow separation of the oral and nasal lining (Figure 1-A). Both markings are connected anteriorly to create round tips of the flaps. The palate is infiltrated with $0.2 \%$ Lidocaine and 1:200,000 Epinephrine. Then, full-thickness relaxing incisions are made through the mucoperiosteum down to the bone of the maxilla. Using a scalpel, an incision along the cleft margin is made, allowing separation of the oral lining from the nasal lining. Mucoperiosteal flaps are elevated by subperiosteal dissection on the maxilla and palatine bone with care to the palatine arteries not to be injured. Then, subperiosteal undermining is carried along the posterior edge of the hard palate to release any attached nasal lining from the hard palate (Figure 1 - B). After wide sub-periosteal undermining on the nasal surface of the palatine bones and maxilla, mobilizing the nasal mucosa of the palatal shelves, the nasal lining and periosteum are reflected medially toward the cleft, facilitating a tension-free closure of the nasal layer (Figure 1- C). Later, both 
mucoperiosteal flaps are elevated with careful muscle dissection and freeing of the levator velii palatini muscles from the posterior edge of the hard palate before posterior repositioning and tension-free approximation in the midline. The two paired levator muscles are sutured to one another with an absorbable suture by horizontal mattress method (Figure 2 -B and $C$ ). Appropriate tension to the repair is important, avoiding an extremely loose or tight muscle bundle that may compromise velar movement. The oral mucosa is closed with absorbable horizontal mattress stitches. Anterior to the muscle repair, the horizontal mattress stitch is used to plicate the oral lining against the nasal lining closure, to obliterate dead space (Figure 1 $-D$ and $E$ ) and (Figure 2 -D).
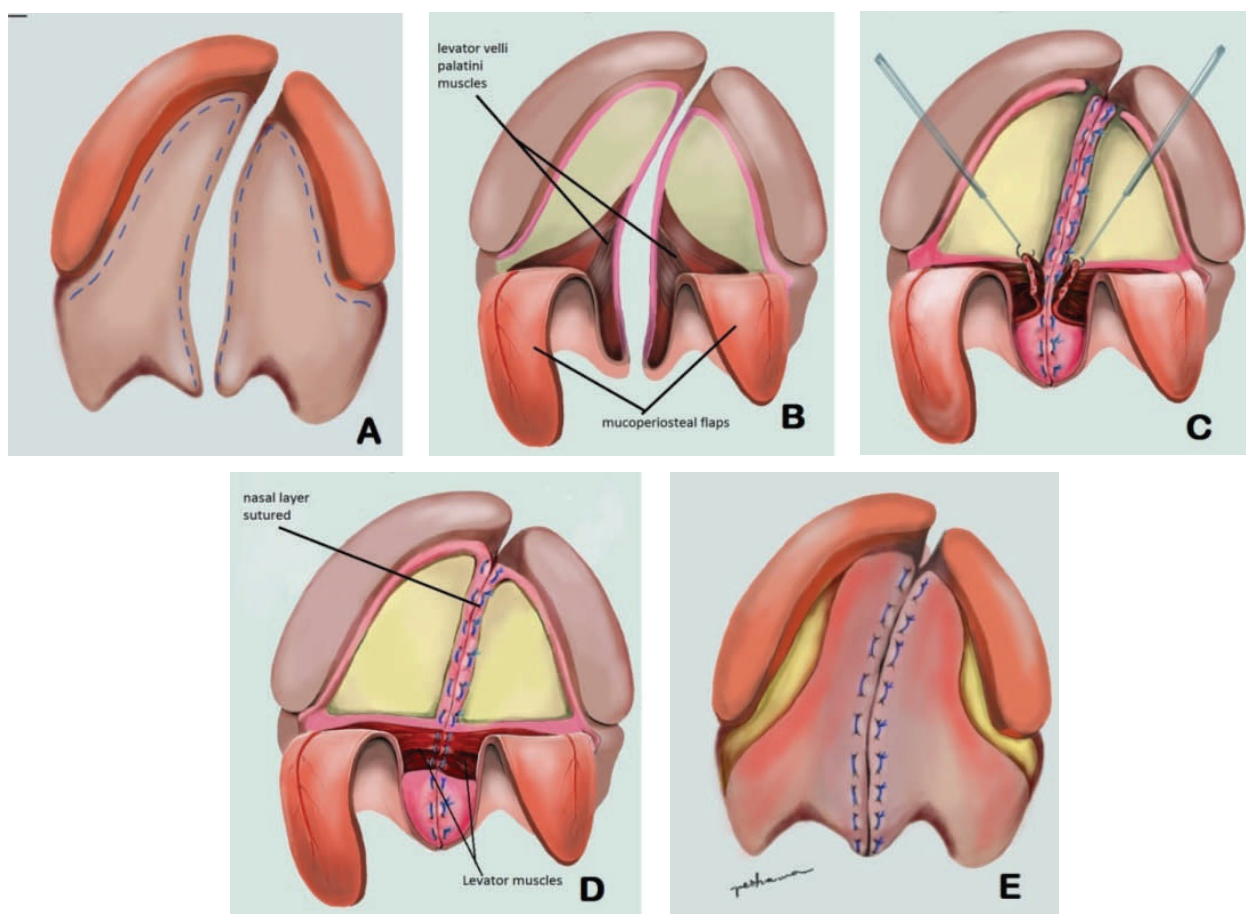

Figure 1: Two - Flaps Palatoplasty with IntravelarVeloplasty. (A) Planning of incisions. (B) mucoperiosteal flaps elevation. (C) release the abnormal Levator muscle attachment. (D) suturing of muscles transversely, and (E) Suturing of the oral layer.
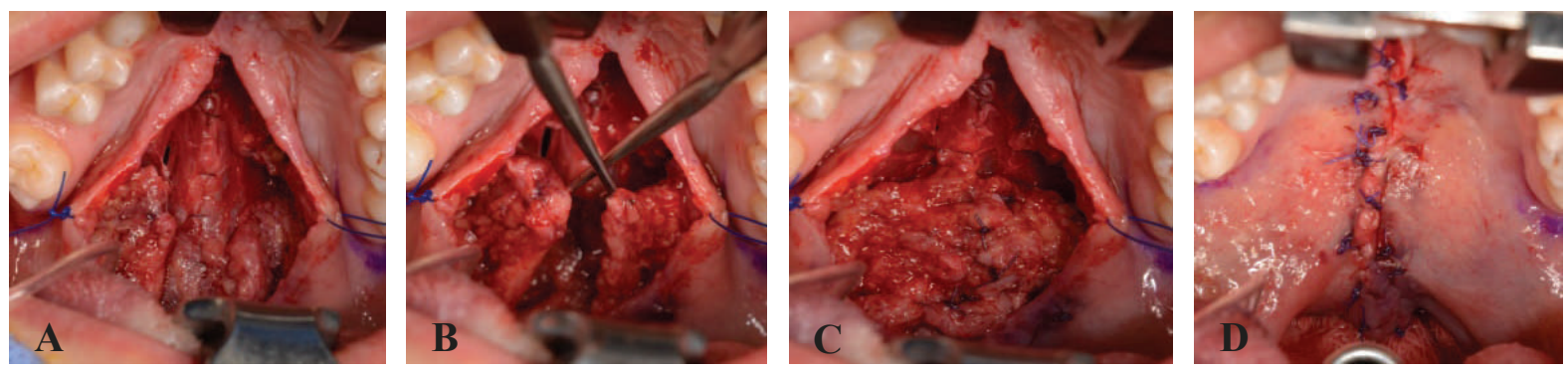

Figure 2: Intravelar veloplasty for a patient with the complete cleft palate. A) Nasal layer sutured, B) Release of Levator velii muscles, C) Muscles sutured in the midline, D) Oral layer sutured. 
Later, follow up with clinical assessment and nasopharyngeal endoscopies were performed. During the clinical check, patients were examined for the presence of any complications (like fistula) and were asked to pronounce certain words and phrases to check the intelligibility and to exclude hyper nasal speech. Then, using a nasopharyngeal endoscope, endoscopy was done to provide information regarding the posterior and superior movement of the velum as well as the medial excursion of the lateral pharyngeal walls during the speech, i.e., velopharyngeal port closure, which was classified into either adequate or inadequate closure.

\section{Ethical Considerations:}

The study protocol was approved by the Research Ethics Committee of College of Medicine, Hawler Medical University. A verbal informed consent was obtained from the parents of each patient $<18$ years old, and from the patient himself if $\geq 18$ years old, before participation in the study. Statistical Analysis:

Data were analyzed using the statistical package for the social sciences (version19). Chi square test of association was used to compare between proportions. When the expected count of more than $20 \%$ of the cells of the table was less than 5 , Fisher's exact test was used. A $P$ value of less or equal to 0.05 was considered statistically significant.

\section{Results}

In this study, 69 patients underwent primary palatal repair and intravelar veloplasty, but 22 patients were discarded for either: losing trace, refusing to be in the study or having secondary palatoplasty already done. The remaining 47 patients were included, 21 male and 26 female patients. Of these 47 patients, 35 had a complete cleft palate, 11 had an incomplete cleft palate, and one patient had a submucous type. The mean age \pm SD was $3.4 \pm 4$ years (range $1-20$ years) at time of repair and mean age \pm SD was $5.8 \pm 5$ years at the time of examination (Table 1).

Table 1: General specifications of the study sample.

\begin{tabular}{llcc}
\hline Characteristics & Categories & No. & $\%$ \\
\hline 1 Gender & Male & 21 & 44.70 \\
& Female & 26 & 55.30 \\
2 Defect type & Complete & 35 & 74.50 \\
& Incomplete & 11 & 23.40 \\
& Submucous & 1 & 2.10 \\
& $\leq 1$ & 19 & 40.40 \\
& $1.1-2$ & 14 & 29.80 \\
& $>2$ & 14 & 29.80 \\
& Total & 47 & 100.00 \\
\hline
\end{tabular}


During the clinical assessment, 33 patients $(70.2 \%)$ could pronounce pharyngeal sounds with or without some degree of hypernasality. On the other hand, a gross hypernasal speech was present in 19 patients $(40.4 \%)$, including patients who got a postoperative oronasal fistula. During the nasopharyngeal endoscopic assessment, patients were grouped according to velar port closure into two groups: adequate and inadequate velar port closure (Table 2). Regarding the postoperative velopharyngeal port assessment, the adequate closure was found in $66 \%$ of the whole sample, the majority of them had an incomplete cleft palate, i.e., $100 \%$ of incomplete CP showed adequate closure while only $54.3 \%$ of complete CP had adequate closure (Table 2). One of the complications encountered was oronasal fistula formation, which appeared in 5 patients $(10.6 \%)$ only, and all of those had a complete cleft palate as the original defect; i.e., $14.3 \%$ of patients with complete cleft palate developed fistulae comparing to $0 \%$ of incomplete and submucous cleft palate patients (Table 2).

Table 2: Association of Defect Type With Postoperative Outcome.

\begin{tabular}{|c|c|c|c|c|c|}
\hline Variable & $\begin{array}{c}\text { Complete } \\
n=35 \\
\text { No.(\%) }\end{array}$ & $\begin{array}{c}\text { Defect Type } \\
\text { Incomplete } \\
n=11 \\
\text { No.(\%) }\end{array}$ & $\begin{array}{c}\text { Submucous } \\
n=1 \\
\text { No.(\%) }\end{array}$ & $\begin{array}{l}\text { Total } \\
\mathrm{n}=47 \\
\text { No.(\%) }\end{array}$ & $P$ value* \\
\hline \multicolumn{6}{|l|}{ VP closure: } \\
\hline Adequate & $19(54.3)$ & $11(100)$ & $1(100)$ & $31(66)$ & \multirow{2}{*}{0.009} \\
\hline Inadequate & $16(45.7)$ & $0(0)$ & $0(0)$ & $16(34)$ & \\
\hline \multicolumn{6}{|c|}{ Hypernasal Speech } \\
\hline Yes & $18(51.4)$ & $1(9.1)$ & $0(0)$ & $19(40.4)$ & \multirow{2}{*}{0.022} \\
\hline No & $17(48.6)$ & $10(90.9)$ & $1(100)$ & $28(59.6)$ & \\
\hline \multicolumn{6}{|c|}{ Pharyngeal sounds } \\
\hline Yes & $21(60)$ & $11(100)$ & $1(100)$ & $33(70.2)$ & \multirow{2}{*}{0.02} \\
\hline No & $14(40)$ & $0(0)$ & $0(0)$ & $14(29.8)$ & \\
\hline \multicolumn{6}{|l|}{ Fistula rate } \\
\hline Yes & $5(14.3)$ & $0(0)$ & $0(0)$ & $5(10.6)$ & \multirow{2}{*}{0.390} \\
\hline No & $30(85.7)$ & $11(100)$ & $1(100)$ & $42(89.4)$ & \\
\hline
\end{tabular}

* Fisher's Exact Test 
The age at time of repair showed significant association with postoperative degree of velopharyngeal closure, as $89.5 \%$ of patient operated at age of 1 year or less, had adequate VP closure and higher percent of ability to make pharyngeal sounds $(94.7 \%, P=0.009)$, while patients operated after two years of age (Figure 3 ) had only $42.9 \%$ adequate VP closure $P=0.01$ (Table 3 ).

Table 3: Association of Operative Age with Postoperative Outcome.

\begin{tabular}{|c|c|c|c|c|c|}
\hline \multirow[b]{2}{*}{ Variable } & \multicolumn{3}{|c|}{ Age at time of operation (year) } & \multirow[b]{2}{*}{$\begin{array}{c}\text { Total } \\
n=47 \\
\text { No.(\%) }\end{array}$} & \multirow[b]{2}{*}{$P$ value } \\
\hline & $\begin{array}{c}\leq 1 \\
n=19 \\
\text { No.(\%) }\end{array}$ & $\begin{array}{c}1.1-2 \\
n=14 \\
\text { No.(\%) }\end{array}$ & $\begin{array}{c}>2 \\
n=14 \\
\text { No. }(\%)\end{array}$ & & \\
\hline \multicolumn{6}{|l|}{ VP Closure: } \\
\hline Adequate & $17(89.5)$ & $8(57.1)$ & $6(42.9)$ & $31(66)$ & \multirow[b]{2}{*}{$0.01^{*}$} \\
\hline Inadequate & $2(10.5)$ & $6(42.9)$ & $8(57.1)$ & 16(34) & \\
\hline \multicolumn{6}{|c|}{ Hypernasal Speech } \\
\hline Yes & $6(31.6)$ & $4(28.6)$ & $9(64.3)$ & $19(40.4)$ & \multirow[b]{2}{*}{0.093} \\
\hline No & $13(68.4)$ & $10(71.4)$ & $5(35.7)$ & $28(59.6)$ & \\
\hline \multicolumn{6}{|c|}{ Pharyngeal Sounds } \\
\hline Yes & $18(94.7)$ & $8(57.1)$ & $7(50)$ & $33(70.2)$ & \multirow[b]{2}{*}{$0.009^{*}$} \\
\hline No & $1(5.3)$ & $6(42.9)$ & $7(50)$ & $14(29.8)$ & \\
\hline \multicolumn{6}{|c|}{ Fistula Rate } \\
\hline Yes & $0(0)$ & $3(21.4)$ & $2(14.3)$ & $5(10.6)$ & \multirow{2}{*}{$0.096^{*}$} \\
\hline No & $19(100)$ & $11(78.6)$ & $12(85.7)$ & $42(89.4)$ & \\
\hline
\end{tabular}

* Fisher's Exact Test
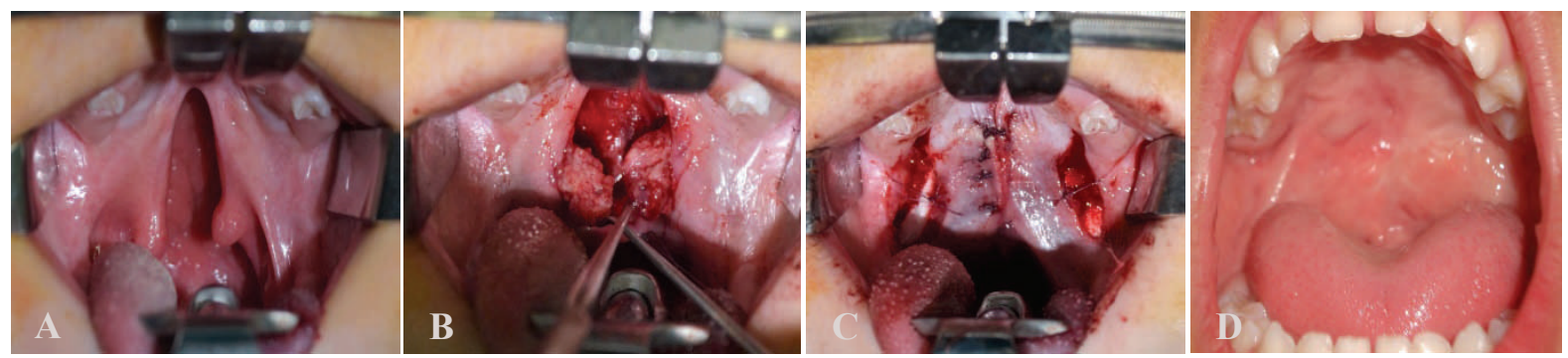

Figure 3: 1.8 year old child with complete CP.(A) Preoperative view (B) Velar muscles released (C) immediate postoperative view. (D) Result after five years with adequate length of velum. 


\section{Discussion}

The velum is known to have a great role in producing intraoral pressure and thereby producing certain sounds by its movement against and down the pharyngeal musculature and maintenance of nasopharyngeal closure during feeding. ${ }^{11}$ That's why we aim to repair the abnormal velar muscle attachment to gain a normal functioning velum. In other words, the success of palatal repair is directly related to the adequacy of velar muscles repair $^{12}$. Veloplasty is a key step in gaining more favorable results in term of velar physiology starting from baby feeding problems elimination and speech improvement later on. ${ }^{9,10}$ This study was a review for patients who had palatoplasty with intravelar veloplasty done by the same surgeon, it showed significant association of defect type with adequacy of velopharyngeal closure $(100 \%$ of incomplete CP showed adequate VP closure, $P<0.009$ ) and generally improved closure in very wide clefts, because VPI is associated with Veau type of cleft and its more common in wide clefts involving more hard palate, that's why incomplete cleft palates show better results after repair. ${ }^{3,5,6}$ The hypernasal speech was also encountered despite velar muscle repair. This could be due to very wide original defect, which was significant statistically (51.4\% of complete CP, $P=0.022$ ). But also speech assessment was affected by factors like personal judgment, patient's satisfaction, and fistula formation especially posterior fistula. A study by Henkle et al ${ }^{13}$ in 2004, stated that repositioning of the velar muscular sling is achieved by intravelar veloplasty with dissection of muscle from the posterior shelf of hard palate with three years follow up. Articulation of pharyngeal sounds like /n/, /l/, /d/ were satisfactory for both groups; control (IVV) and (IVV with wave line technique), as in our study $70.2 \%$ of patients could fairly pronounce pharyngeal sounds, while the rest $29.8 \%$ who couldn't, most of them were the sample age extremities; the young for having mile stone still not developed well (the youngest patient was 2 years old at time of examination), and the older age group for delay presentation or having other disabilities or associated syndrome. Mentioning the age; patient with delayed presentation had the highest percent of hypernasal speech $(64.3 \%$ of patients $>2$ years old had hypernasal speech, $P=0.093$ ) and lowest percent for ability to pronounce pharyngeal sounds comparing to the younger age groups (50\% couldn't make pharyngeal sounds, $P=0.009)$. Ha $S$ et $\mathrm{al}^{14}$ in 2015 obtained a result of only $28.8 \%$ total of hypernasality as they used IVV with different types of palatoplasty, while in our study, $59.6 \%$ had no hypernasality and $70.2 \%$ could pronounce pharyngeal plosive sounds, as mentioned above, however we couldn't obtain precise speech assessment as we lack a speech therapist or speech therapy center in Erbil. Similarly, Talmant $\mathrm{JC}^{15}$ in 2006 had cut the doubt of the important role of IVV on the function of soft palate during the speech, claiming a significant improvement of his patients' speech results after using IVV as a functional approach for palatoplasty. Oronasal fistula rate in our study was $10.6 \%$ most of which were the anterior type and too small, i.e., Not affecting the speech or feeding unless under pressure, while big posterior fistula (which appear in two patients) caused hypernasality and inability to hold pressure in the oral cavity. Regardless the fistula size, all five patients were scheduled for fistula repair. During VP port assessment, the adequate velar closure was significantly associated with early repair $(89.5 \%$ of patients $\leq 1$ year showed adequate closure, $P=0.01)$.In the other hand, most patients with VPI were those with delay presentation (patients >two years old at the time of palatoplasty had $57.1 \%$ inadequate VP closure, $P=0.01$ ). The VPI rate $(34 \%)$ was close to the one obtained in a study done by Fattah and $\mathrm{Ali}^{12}$ in 2015 in Erbil who included more than one technique for palatoplasty. 
Inadequate VP closure could be part of VP dysfunction (VPD) as the normal function of Velo-pharynx requires not only Velum closure but proper coordination and speed of closure as well as a reopening appropriate for the specific task. ${ }^{6}$ Therefore, children showing defective speech should get speech therapy which will aid to rehabilitate the compensatory mal-articulation and abnormal phoneme production. Operating on the adult patient has many challenges including wide clefts, adherent mucoperiosteal flaps due to chronic inflammatory process related to poor hygiene and/or smoking. Late effect of age is mainly related to mal-articulation with or without poor velopharyngeal function. In a study done in India by Murthy $^{16}$ in 2009 for primary palatal repair in adult patients (sample included children more than 10 years old), the author chose two flaps palatoplasty with IVV in patient with complete cleft palate and he suggested that it was superior to pharyngeal flap in term of complications with less VPI but no much statistical significance for detailed speech outcome, as speech is affected by much more factors for articulation other than the velum, however IVV was done even when using other techniques; like Langenbeck or $\mathrm{V}-\mathrm{Y}$ push back for incomplete CP. ${ }^{16}$ Murthy records $55 \%$ improvement in intelligibility postoperatively compared to only $22 \%$ preoperatively, which was attributed to significant improvement in articulation and resonance and overall improvement in speech parameters. While in our study, improvement of speech was clearly noticed by patient's family, but we didn't include family's satisfaction as a variable, as it would be affected by many factors as family's education, original defect, quality of speech before the repair, and age of the patient. Achieving normal speech was difficult, except in few cases of incomplete clefts. However, this study had many limitations in term of a detailed evaluation of speech production, as hypernasality could be due to more than one component of articulation parts like alveolus, lips, tongue and teething. ${ }^{11}$

\section{Conclusion}

Intravelar veloplasty significantly improves velopharyngeal port closure and speech production later in life. Velar port closure is highly affected by cleft palate type, thus best closure achieved with submucous and incomplete cleft palate. Early repair of cleft palate, before $1^{\text {st }}$ year of age, yield best results in regard to adequate velar port closure, less hypernasality of speech, and better development of speech articulation. We recommend performing intravelar veloplasty as a routine step during cleft palate repair, whatever the cleft palate type is, and whatever palatoplasty technique is chosen, because even the mildest incomplete cleft palates are known to have some degree of abnormal muscle attachment. Team work is needed to evaluate, treat, and follow up patients with cleft palate especially speech therapy institute is lacking in Erbil. A detailed prospective study with long term follow up will yield better statistics.

\section{Competing interests}

The authors declare that they have no competing interests.

\section{References}

1. Hopper RA. Cleft Lip and Palate: Embryology, Principles, and Treatment. In: Thorn $\mathrm{CH}$ (editor). Grabb and Smith plastic Surgery: $7^{\text {th }}$ ed. Philadelphia: Lippincott Williams \& Wilkins; 2013. P. 173-99.

2. Leow AK, Lo LJ. Palatoplasty: Evolution and Controversies. Chang Gung Med J 2008; 31(4):335-45.

3. Georgiade GS. Plastic, Maxillofacial, and reconstructive surgery. $3^{\text {rd }}$ ed. Baltimore: Williams and Wilkins; 1997.

4. Barbosa DA, Scarmagnani RH, Fukushiro AP, Trindade IE, Yamashita RP. Surgical Outcome of Pharyngeal flap surgery and Intravelar veloplasty on the velopharyngeal Function. CoDAS 2013; 25(5):451-5.

5. Marsh JL, Grames LM, Holtman B. Intravelar Veloplasty: A Prospective Study. Cleft Palate J 1989; 1(26):46-9.

6. Marsh JL. Velo-Pharyngeal Dysfunction: Evaluation and Management. Indian J PlastSurg 2009; 42(suppl):S129-36. 
7. Jarvis BL, Trier WC. The effect of Intravelar Veloplasty on Velopharyngeal Competence following Pharyngeal Flap Surgery. Cleft Palate J 1988; 25(4):389-93.

8. Agrawal K. Cleft Palate Repair and Variations. Indian J Plast Surg 2009; 42(suppl): S102-9.

9. Nahai FR, Williams JK, Burstein FD, Martin J, Thomas J. The Management of Cleft Lip and Palate: Pathways for Treatment and Longitudinal Assessment. Seminars in Plastic Surgery 2005; 19(4):275-85.

10. Andrades $\mathrm{P}$, Monteros AE, Shell $\mathrm{DH}$, Thurston TE, Fowler jS, Xavier ST, et al. The Importance of Radical Intravelar Veloplasty during Two- Flaps Palatoplasty. Plast Reconstr Surg 2008; 122(4):1121-30.

11. Kummer AW. Cleft palate and Crainiofacial anomalies: Effect on speech and resonance: $2^{\text {nd }}$ ed. New York: Delmar Healthcare; 2008.

12. Fattah JH, Ali HS. Evaluation of cleft lip and palate management in Erbil. Zanco J Med Sci 2015;19(1):866-73.

13. Henkel KO, Dieckmann A, Dieckmann O, Lenz JH, Gundlach KKH. Veloplasty Using TheWave-Line Technique Versus Classic Intravelar Veloplasty. Cleft Palate Craniofac J 2004;41(1).

14. Ha S, Koh KS, Moon $\mathrm{H}$, Jung $\mathrm{S}$, Oh TS. Clinical Outcomes of Primary Palatal Surgery in Children with Non-Syndromic Cleft Palate with or without Lip. BioMed Research International 2015; 2015:185459.

15. Talmant JC. Evolution of the Functional Repair Concept for Cleft Lip and Palate Patients. Indian J PlasSurg 2006; 39(2):196-209.

16. Murthy J. Management of Cleft Lip and Palate in Adults. Indian J PlastSurg 2009; 42(suppl):S11622. 